\title{
Averages of exponential twists of the Liouville function
}

\author{
M. Ram Murty ${ }^{1}$ and A. Sankaranarayanan \\ (Communicated by Peter Sarnak)
}

\begin{abstract}
Let $\lambda(n)$ denote the Liouville function and consider the sum $S(x, \alpha)=$ $\sum_{n \leq x} \lambda(n) e^{2 \pi i n \alpha}$. We prove that for all $\alpha$ of irrational type 1 (that includes all algebraic irrationalities) $S(x, \alpha)=O\left(x^{4 / 5+\varepsilon}\right)$ for any $\varepsilon>0$. The method is extended to study more general sums of the form $\sum_{n \leq x} a(n) e^{2 \pi i n \alpha}$ for a general class of arithmetical functions $a(n)$. The main technique is the Vinogradov-Vaughan method of studying exponential sums.
\end{abstract}

2000 Mathematics Subject Classification: 11L07; 11N36.

\section{Introduction}

Pólya suggested the following approach to the Riemann hypothesis. Let $\lambda(n)$ be the Liouville function which is the completely multiplicative function defined as $\lambda\left(p^{\alpha}\right)=$ $(-1)^{\alpha}$ for prime powers $p^{\alpha}$. Now consider the sum,

$$
S(x)=\sum_{n \leq x} \lambda(n)
$$

Pólya $[\mathrm{P}]$ conjectured that $S(x) \leq 0$ for $x \geq 2$ and noted that if this conjecture is true, then the Riemann hypothesis follows. Indeed, we have

$$
\sum_{n=1}^{\infty} \frac{\lambda(n)}{n^{s}}=\frac{\zeta(2 s)}{\zeta(s)}
$$

and by partial summation,

$$
\frac{\zeta(2 s)}{\zeta(s)}=s \int_{1}^{\infty} \frac{S(x)}{x^{s+1}} d x .
$$

\footnotetext{
${ }^{1}$ Research partially supported by NSERC and a Killam Research Fellowship.
} 
Thus, by a well-known theorem of Landau (see for example, [EM, p. 132]), we deduce that the left hand side converges for $\operatorname{Re}(s)>a$ where $a$ is the first real singularity of $\zeta(2 s) / \zeta(s)$. Since $\zeta(s)$ has no real zeros, we see that the first real singularity occurs at $s=1 / 2$. Hence, $\zeta(2 s) / \zeta(s)$ converges for $\operatorname{Re}(s)>1 / 2$. Therefore, $\zeta(s) \neq 0$ for $\operatorname{Re}(s)>1 / 2$.

In 1958, Haselgrove $[\mathrm{H}]$ disproved the Pólya conjecture. The smallest counter-example is $x=906,150,257$ for which $S(x)=1$. Lehman [L] later found $S(906,400,000)=$ 708. It can be shown that there are infinitely many counter-examples. (See for example, Anderson-Stark [AS].)

There is good reason to resurrect Pólya's approach. In fact, in $[\mathrm{Mu}]$ an elliptic analogue of the Pólya conjecture is proposed and some heuristic and numerical evidence are presented that predict its truth in the case of curves whose Mordell-Weil rank is at least 4 .

Indeed, if $E$ is an elliptic curve over $\mathbb{Q}$ of Mordell-Weil rank at least 4 and $L_{E}(s)=$ $\sum_{n=1}^{\infty} a_{n} / n^{s}$ is the $L$-series of the elliptic curve, then the Riemann hypothesis and the Birch and Swinnerton-Dyer conjectures suggest that

$$
\sum_{n \leq x} a_{n} \lambda(n) \geq 0
$$

for sufficiently large $x$. This conjecture is studied in greater detail in $[\mathrm{Mu}]$. In this paper, we focus on $S(x)$ and its "exponential twists" (defined below).

Suppose now that we consider the hypothesis that for some $\theta<1$,

$$
S(x)=O\left(x^{\theta}\right)
$$

Then, the above argument easily yields that $\zeta(s) \neq 0$ for $\operatorname{Re}(s)>\theta$, which we refer to as a quasi-Riemann hypothesis. In fact, the Riemann hypothesis is equivalent to the assertion that (1) holds for every $\theta>1 / 2$.

If instead of averages of the Liouville function, we consider the sum

$$
S\left(x, \frac{a}{q}\right):=\sum_{n \leq x} \lambda(n) \mathbf{e}(n a / q)
$$

where $\mathbf{e}(t)=e^{2 \pi i t}$, then the estimate

$$
S\left(x, \frac{a}{q}\right)=O\left(x^{\theta}\right)
$$

is equivalent to the statement that the Dirichlet $L$-functions, $L(s, \chi)$ where $\chi$ is any primitive character $\bmod q$, has no zeros in the half-plane $\operatorname{Re}(s)>\theta$. To see this, note that using Gauss sums, we may write 


$$
S(x, \chi):=\sum_{n \leq x} \lambda(n) \bar{\chi}(n)=\frac{1}{\tau(\chi)} \sum_{a \bmod q} \chi(a) S\left(x, \frac{a}{q}\right)
$$

from which we see that the Dirichlet series

$$
\sum_{n=1}^{\infty} \frac{\lambda(n) \chi(n)}{n^{s}}
$$

converges for $\operatorname{Re}(s)>\theta$. Since,

$$
\frac{L(2 s, \chi)}{L(s, \chi)}=s \int_{1}^{\infty} \frac{S(x, \bar{\chi})}{x^{s+1}} d x
$$

we deduce that $L(s, \chi)$ has no zeros for $\operatorname{Re}(s)>\theta$.

Motivated by these considerations, it seems natural to consider the exponential sum:

$$
S(x, \alpha):=\sum_{n \leq x} \lambda(n) \mathbf{e}(n \alpha)
$$

for any real $\alpha$. The estimates we obtain for such sums depend on the type of $\alpha$. Let us recall this notion (see [KN, p. 121ff] for more details). Let $\psi$ be a non-decreasing positive function that is defined at least for all positive integers. The irrational number $\alpha$ is said to be of type $<\psi$ if $q\|q \alpha\| \geq 1 / \psi(q)$ holds for all positive integers $q$. If $\psi$ is a constant function, then an irrational $\alpha$ of type $<\psi$ is also called of constant type. Let $\eta$ be a positive real number or infinity. The irrational number $\alpha$ is said to be of type $\eta$ if $\eta$ is the supremum of all $\gamma$ for which

$$
\liminf _{q \rightarrow \infty} q^{\gamma}\|q \alpha\|=0
$$

where $q$ runs through the positive integers.

The relationship between these two definitions is that an irrational number $\alpha$ is of type $\eta$ if and only if for every $\tau>\eta$ there is a constant $c=c(\tau, \alpha)$ such that $\alpha$ is of type $<\psi$ where $\psi(q)=c q^{\tau-1}$. (See $\left.[\mathrm{KN}, \mathrm{p} .121]\right)$

A celebrated theorem of Khintchine (see for example [La, p. 23]) states that if $\Psi(q)$ is a positive function such that

$$
\sum_{q=1}^{\infty} \Psi(q)
$$

converges, then for almost all $\alpha$,

$$
\|q \alpha\| \gg \Psi(q)
$$


for all $q$ sufficiently large. If

$$
\sum_{q=1}^{\infty} \Psi(q)
$$

diverges, then for almost all $\alpha$, there exist infinitely many $q$ such that

$$
\|q \alpha\| \leq \Psi(q)
$$

Therefore, almost all numbers are of type 1. By virtue of Roth's theorem, we know that all algebraic irrationalities $\alpha$ satisfy

$$
\left|\alpha-\frac{p}{q}\right| \gg \frac{1}{q^{2+\varepsilon}} .
$$

Hence all algebraic irrationalities are of type 1.

Our goal in this paper is to prove:

Theorem 1. For all $\alpha$ of type 1 (which necessarily includes all algebraic irrationalities), we have

$$
S(x, \alpha)=O\left(x^{4 / 5+\varepsilon}\right)
$$

for any $\varepsilon>0$.

Observe that by Parseval's formula, we have

$$
\int_{0}^{1}|S(x, \alpha)|^{2} d \alpha=x+O(1)
$$

so that it is reasonable to expect that for any $\varepsilon>0, S(x, \alpha)=O\left(x^{1 / 2+\varepsilon}\right)$ for almost all $\alpha$. Indeed, if we let

$$
E(x, \delta)=\left\{\alpha:|S(x, \alpha)|>x^{1 / 2+\delta}\right\}
$$

then $E(x, \delta)$ is measurable and Parseval's formula gives that its measure cannot exceed $x^{-2 \delta}$.

As pointed out to us by J. Oesterlé, one can use Carleson's theorem [C] to deduce more. Recall that the celebrated theorem of Carleson says that if

$$
\sum_{k=0}^{\infty}\left|c_{k}\right|^{2}<\infty
$$


then the Fourier series

$$
\sum_{k=0}^{\infty} c_{k} \mathbf{e}(k \theta)
$$

converges for almost all $\theta \in \mathbb{R}$. If $\sum_{k=0}^{\infty} b_{k}$ is convergent, and $f(n)$ is an increasing function, then by partial summation, it is easily seen that

$$
\sum_{k \leq n} b_{k} f(k)=o(f(n))
$$

as $n \rightarrow \infty$. Indeed, given $\varepsilon>0$, choose $n_{0}$ such that $\left|\sum_{k=m}^{n} b_{k}\right| \leq \varepsilon$ for $n \geq m \geq n_{0}$. Then,

$$
\sum_{k=n_{0}}^{n} b_{k} f(k)=A(n) f(n)-\sum_{k=n_{0}}^{n-1} A(k)(f(k+1)-f(k))
$$

where $A(k)=\sum_{j=n_{0}}^{k} b_{j}$. The right hand side is clearly $\ll \varepsilon f(n)$ from which the result is immediate. Now if $a_{k}$ is a sequence of complex numbers of absolute value 1 , and $S_{n}(x)=\sum_{k=0}^{n} a_{k} \mathbf{e}(x)$, then setting $f(x)=x^{1 / 2+\varepsilon}$, then

$$
\sum_{k=1}^{\infty} 1 /|f(k)|^{2}<\infty
$$

so that by Carleson's theorem,

$$
\sum_{k=1}^{\infty} \frac{a_{k}}{f(k)} \mathbf{e}(k \theta)
$$

converges almost everywhere. Thus, the partial sums satisfy

$$
\sum_{k \leq x} a_{k} \mathbf{e}(k \theta) \ll O\left(x^{1 / 2+\varepsilon}\right)
$$

for almost all $\theta$. It is clear that this result holds for $a_{k}=O\left(k^{\varepsilon}\right)$ as well. Of course, Carleson's theorem gives us no idea to which $\theta$ the result applies.

The above argument can be refined in the obvious way. In fact, we can take $f(x)=$ $x^{1 / 2}(\log x)^{1 / 2+\varepsilon}$ to deduce

$$
\sum_{k \leq x} a_{k} \mathbf{e}(k \theta)=O\left(x^{1 / 2}(\log x)^{1 / 2+\varepsilon}\right)
$$

for almost all $\theta$ whenever

$$
\sum_{k=1}^{\infty} \frac{\left|a_{k}\right|^{2}}{|f(k)|^{2}}<\infty
$$


It seems that Davenport [D] was the first to consider the sums $S(x, \alpha)$ and the cognate sums:

$$
M(x, \alpha):=\sum_{n \leq x} \mu(n) e(n \alpha)
$$

where $\mu$ denotes the Möbius function. Davenport proves that for every $\alpha, M(x, \alpha)=$ $O\left(x /(\log x)^{A}\right)$ for any $A>0$ uniformly in $\alpha$. At the end of his paper, he remarks that a similar treatment works for $S(x, \alpha)$. See also the related paper of Bateman and Chowla $[\mathrm{BC}]$.

In a paper of Hajela and Smith [HS], they also investigate the sum $M(x, \alpha)$ and obtain various improvements of the results of Davenport. For instance, they show that $M(x, \alpha)=O\left(x \exp \left(-c(\log x)^{1 / 2}\right)\right)$ for some sufficiently small $c>0$ under the assumption that none of the $L(s, \chi)$ have Siegel zeros. If the generalised Riemann hypothesis holds, they prove that $M(x, \alpha)=O\left(x^{5 / 6+\varepsilon}\right)$. Baker and Harman $[\mathrm{BH}] \mathrm{im}-$ proved the Hajela-Smith exponent of $5 / 6$ to $3 / 4$ under the same hypothesis. We will show below (see Corollary 4 ) that $M(x, \alpha)=O\left(x^{4 / 5+\varepsilon}\right)$ for all $\alpha$ of type 1 .

Our main tool is Vaughan's method which we outline in section 2. It is applicable, in a wider context. In fact, in the final section of the paper, we show that one can expect an estimate of the type

$$
\sum_{n \leq x} c(n) \mathbf{e}(n \alpha) \ll x^{\psi}
$$

for all $\alpha$ of type 1 and $\psi<1$ for a wide class of functions $c(n)$ satisfying the estimate $c(n)=O\left(n^{\varepsilon}\right)$. More precisely:

Theorem 2. Suppose that for two functions $a$ and $b$ satisfying $a(n)=O\left(n^{\varepsilon}\right)$ and $b(n)=$ $O\left(n^{\varepsilon}\right)$, there exist $\theta<1$ and $\phi<1$ respectively such that for all $\alpha$ of type 1 ,

$$
\begin{aligned}
& \sum_{n \leq x} a(n) \mathbf{e}(n \alpha) \ll x^{\theta} \min \left(1, \frac{1}{\|\alpha\|}\right) \\
& \sum_{n \leq x} b(n) \mathbf{e}(n \alpha) \ll x^{\phi} \min \left(1, \frac{1}{\|\alpha\|}\right)
\end{aligned}
$$

where $\|\alpha\|$ denotes the distance of $\alpha$ to the nearest integer. Then, there is a $\psi<1$ such that

$$
\sum_{n \leq x} c(n) \mathbf{e}(n \alpha) \ll x^{\psi} \min \left(1, \frac{1}{\|\alpha\|}\right)
$$

where $c(n)$ is defined by the recursion 


$$
a(n)=\sum_{d \mid n} b(d) c(n / d)
$$

This result has an interesting algebraic interpretation. If we consider the set of Dirichlet series $\sum_{n=1}^{\infty} a(n) / n^{s}$ satisfying (3), with $a(1) \neq 0$, then the collection is an abelian group under multiplication of Dirichlet series.

In the special case $B(s)=\zeta(s)$, one can derive more precise results:

Theorem 3. Let $\sum_{n=1}^{\infty} c(n) / n^{s}=A(s) / \zeta(s)$ where $A(s)=\sum_{n=1}^{\infty} a(n) / n^{s}$. Suppose that (3) is satisfied. Then, for all $\alpha$ of type 1 ,

$$
\sum_{n \leq x} c(n) \mathbf{e}(n \alpha) \ll x^{4 / 5+\varepsilon}+x^{(2+3 \theta) / 5+\varepsilon} .
$$

Let us observe that in Theorem 3, the $x^{4 / 5+\varepsilon}$ dominates whenever $\theta<2 / 3$.

One cannot expect the result in Theorem 3 to hold for all $\alpha$. Indeed, if $\alpha=a / q$ is a rational number with $(a, q)=1$, then, in the special case $A(s)=-\zeta^{\prime}(s)$, we have

$$
\sum_{n \leq x} \Lambda(n) \mathbf{e}\left(n \frac{a}{q}\right) \sim \frac{\mu(q)}{\phi(q)} x
$$

as $x \rightarrow \infty$. A similar remark applies to Theorem 2. One does not, however, expect this phenomenon to persist for the exponential twists of the Liouville function or the Möbius function.

Corollary 4. For all $\alpha$ of type 1,

$$
\sum_{n \leq x} \mu(n) \mathbf{e}(n \alpha) \ll x^{4 / 5+\varepsilon}
$$

The following corollary is of course implicit in [D, p. 143].

Corollary 5. Let $\Lambda(n)$ denote the usual von Mangoldt function. Then, for all $\alpha$ of type 1 ,

$$
\sum_{n \leq x} \Lambda(n) \mathbf{e}(n \alpha) \ll x^{4 / 5+\varepsilon}
$$

If $A(s)=\zeta_{K}(s)$, the Dedekind zeta function of a number field $K$, then Chandrasekharan and Narasimhan $[\mathrm{CN}]$ have shown that

$$
\sum_{n \leq x} a(n) \mathbf{e}(n \alpha) \ll x^{\theta}
$$

for some $\theta<1$ whenever $K$ is a quadratic extension of $\mathbb{Q}$. This leads us to the following application: 
Corollary 6. If $a(n)$ denotes the number of integral ideals of norm $n$ in a quadratic field $K$, and

$$
c(n)=\sum_{d \mid n} \mu(d) a(n / d)
$$

then for all $\alpha$ of type 1 ,

$$
\sum_{n \leq x} c(n) \mathbf{e}(n \alpha) \ll x^{\psi}
$$

with $\psi<1$.

The interest in Corollary 6 is that it is expected to be true for any field $K$. In fact, it is related to a classical conjecture of Dedekind which predicts that $\sum_{n=1}^{\infty} c(n) / n^{s}$ extends to an entire function if $c(n)$ is defined as above and $a(n)$ is the number of integral ideals of norm $n$ in the field $K$. The Riemann hypothesis for Dedekind zeta functions would imply that $\sum_{n \leq x} c(n)=O\left(x^{1 / 2+\varepsilon}\right)$. However, this is not known. In fact, it is not even known if the above sum is $O\left(x^{\theta}\right)$ for some $\theta<1$ for a general number field $K$.

There seem to be two results in the literature aiming to treat general sums of the form (3). The first is a paper of Daboussi and Delange [DD] which shows that

$$
\sum_{n \leq x} f(n) \mathbf{e}(n \alpha)=o(x)
$$

as $x \rightarrow \infty$ for $\alpha$ irrational and $f$ multiplicative satisfying

$$
\sum_{n \leq x}|f(n)|^{2}=O(x)
$$

See also the related work of Goubin $[\mathrm{G}]$ and Delange [De]. The second result is due to Montgomery and Vaughan [MV] where they show that for almost all $\alpha$ including algebraic irrationalities,

$$
\sum_{n \leq x} f(n) \mathbf{e}(n \alpha) \ll \frac{x}{\log x}
$$

for any multiplicative function $f$ satisfying $|f(n)| \leq 1$. Theorem 2 can be seen as a variation of this theme and an extension of the result of Montgomery and Vaughan [MV].

There are several other sporadic results for special arithmetical functions, scattered in the literature. We will cite a few exemplary ones. For instance, Chowla $[\mathrm{Ch}]$ proved that for all irrational $\alpha$,

$$
\sum_{n \leq x} d(n) \mathbf{e}(n \alpha)=o(x \log x)
$$


as $x \rightarrow \infty$, where $d(n)$ denotes the number of divisors of $n$. Erdös [E] proved that

$$
\sum_{n \leq x} d(n) \mathbf{e}(n \alpha)=O\left(x^{1 / 2} \log x\right)
$$

for almost all $\alpha$. Our remarks concerning the application of Carleson's theorem would give (using a standard Tauberian argument to derive the necessary asymptotics)

$$
\sum_{n \leq x} d(n) \mathbf{e}(n \alpha)=O\left(x^{1 / 2}(\log x)^{2+\varepsilon}\right)
$$

for almost all $\alpha$ which is only slightly weaker than the result of Erdös. In the same paper, Erdös $[\mathrm{E}]$ proves that if $r(n)$ denotes the number of ways of writing $n$ as a sum of two squares, then

$$
\sum_{n \leq x} r(n) \mathbf{e}(n \alpha)=O\left(x^{1 / 2} \log x\right)
$$

for almost all $\alpha$. Here, however, our remarks give an improvement. Indeed, by applying Carleson's theorem as indicated above, we get

$$
\sum_{n \leq x} r(n) \mathbf{e}(n \alpha)=O\left(x^{1 / 2}(\log x)^{1 / 2+\varepsilon}\right)
$$

for almost all $\alpha$ using standard results in analytic number theory. We leave the details as an exercise to the reader.

Acknowledgements. The first author would like to thank the Tata Institute of Fundamental Research for its hospitality during his visit there in January 1999 at which time this work was done. Both authors would like to thank J. Oesterlé for his comments on a preliminary version of this paper as well as Michael Reid for doing some calculations related to $S(x)$.

\section{Vaughan's method}

We begin with a description of Vaughan's method which has its roots in earlier work of I. M. Vinogradov on the Goldbach conjecture. This is exposed for example in [D]. For any $A, B \neq 0$ and $F, G$, we have the formal identity:

$$
\begin{aligned}
\frac{A}{B} & =(1-B G) \frac{A}{B}+A G \\
& =(1-B G)\left(F+\left(\frac{A}{B}-F\right)\right)+A G \\
& =F-B G F+A G+\left(\frac{A}{B}-F\right)(1-B G) .
\end{aligned}
$$


Now let

$$
\begin{array}{ll}
A(s)=\sum_{n=1}^{\infty} \frac{a(n)}{n^{s}} & B(s)=\sum_{n=1}^{\infty} \frac{b(n)}{n^{s}} \\
\frac{A(s)}{B(s)}=\sum_{n=1}^{\infty} \frac{c(n)}{n^{s}} \quad \frac{1}{B(s)}=\sum_{n=1}^{\infty} \frac{\tilde{b}(n)}{n^{s}} \\
F(s)=\sum_{n \leq U} \frac{c(n)}{n^{s}} & G(s)=\sum_{n \leq V} \frac{\tilde{b}(n)}{n^{s}}
\end{array}
$$

be defined for $\sigma=\operatorname{Re}(s) \geq \sigma_{0}$. Here $U$ and $V$ are arbitrary parameters to be chosen later satisfying $U, V \geq 1$.

Lemma 1. We have

$$
c(n)=a_{1}(n)+a_{2}(n)+a_{3}(n)+a_{4}(n)
$$

where

$$
\begin{aligned}
& a_{1}(n)= \begin{cases}c(n) \quad \text { for } n \leq U \\
0 \quad \text { otherwise }\end{cases} \\
& a_{2}(n)=-\sum_{\substack{d e r=n \\
e \leq V, r \leq U}} b(d) \tilde{b}(e) c(r) \\
& a_{3}(n)=\sum_{\substack{d e=n \\
e \leq V}} a(d) \tilde{b}(e) \\
& a_{4}(n)=-\left(\sum_{\substack{d e=n \\
d>U, e>V}} c(d)\left(\sum_{\substack{t r=e \\
r \leq V}} b(t) \tilde{b}(r)\right)\right)
\end{aligned}
$$

Proof. This follows easily from the formal identity indicated above and we refer the reader to Davenport [Da, p. 139] for details.

Lemma 2. We have

$$
\lambda(n)=a_{1}(n)+a_{2}(n)+a_{3}(n)+a_{4}(n)
$$

where

$$
\begin{gathered}
a_{1}(n)= \begin{cases}\lambda(n) & \text { if } n \leq U \\
0 & \text { otherwise }\end{cases} \\
a_{2}(n)=-\sum_{\substack{d e r=n \\
e \leq V, r \leq U}} \mu(e) \lambda(r)
\end{gathered}
$$




$$
\begin{aligned}
& a_{3}(n)=\sum_{\substack{d^{2} e=n \\
e \leq V}} \mu(e) \\
& a_{4}(n)=-\sum_{\substack{d e=n \\
d>U, e>V}} \lambda(d)\left(\sum_{\substack{t r=e \\
r \leq V}} \mu(r)\right) .
\end{aligned}
$$

Proof. The proof is immediate from Lemma 1 upon taking $A(s)=\zeta(2 s), B(s)=\zeta(s)$, $F(s)=\sum_{n \leq U} \lambda(n) / n^{s}, G(s)=\sum_{n \leq V} \mu(n) / n^{s}$ and noticing that $\tilde{b}(n)=\mu(n), b(n)=$ $1, c(n)=\lambda(n)$ and $a(n)=1$ if $n$ is a square and zero otherwise.

\section{Estimates for exponential subsums}

We denote by $\|u\|$ the distance of $u$ from the nearest integer. Let

$$
S_{i}(x)=\sum_{n \leq x} a_{i}(n) \mathbf{e}(n \alpha)
$$

where $a_{i}(n)$ for $i=1,2,3,4$ are the functions defined in Lemma 2.

Erdös $[\mathrm{E}]$ proved that for almost all $\alpha$

$$
\sum_{m=1}^{n} \frac{1}{m\|m \alpha\|}=(1+o(1)) \log ^{2} n .
$$

By partial summation, we deduce that for almost all $\alpha$,

$$
\sum_{m=1}^{n} \frac{1}{\|m \alpha\|} \ll n(\log n)^{3}
$$

If we know the type of $\alpha$, more precise results can be obtained as the following lemma shows.

Lemma 3. Let $\alpha$ be of type $<\psi$. Then,

$$
\sum_{m=1}^{n} \frac{1}{m\|m \alpha\|} \ll \psi(2 n) \log n+\sum_{m=1}^{n} \frac{\psi(2 n) \log n}{n} .
$$

In particular, if $\alpha$ is of constant type, then the sum is $O\left(\log ^{2} n\right)$. If $\alpha$ is of type $\eta$, then the sum above is $O\left(n^{\eta-1+\varepsilon}\right)$. Also,

$$
\sum_{m=1}^{n} \frac{1}{\|m \alpha\|}=O\left(n^{1+\varepsilon}\right)
$$

for $\alpha$ of type 1 . 
Proof. See Lemma 3.3 in [KN, p. 123]. The last assertion follows from partial summation of the estimate for the previous sum.

We now begin our estimation of the sums $S_{i}(x)$ for $i=1,2,3,4$.

Lemma 4. We have

$$
\left|S_{1}(x)\right| \leq U
$$

Proof. This is clear.

Lemma 5. For all $\alpha$ of type 1, we have

$$
S_{2}(x) \ll(U V)^{1+\varepsilon}
$$

for any $\varepsilon>0$.

Proof. We have

$$
\begin{aligned}
S_{2}(x) & =-\sum_{n \leq x}\left(\sum_{\substack{d e r=n \\
e \leq V, r \leq U}} \mu(e) \lambda(r)\right) \mathbf{e}(\operatorname{der} \alpha) \\
& =-\sum_{t \leq U V} \sum_{\substack{e r=t \\
e \leq V, r \leq U}} \mu(e) \lambda(r) \sum_{d \leq x / t} \mathbf{e}(d t \alpha) .
\end{aligned}
$$

Observe that the inner sum is a geometric series, so that

$$
\left|\sum_{d \leq x / t} \mathbf{e}(d t \alpha)\right|=\left|\frac{1-\mathbf{e}(x \alpha)}{1-\mathbf{e}(t \alpha)}\right| \ll \frac{1}{\|t \alpha\|} .
$$

Thus, by Lemma 3, we obtain

$$
S_{2}(x) \ll \sum_{t \leq U V} d(t)\|t \alpha\|^{-1} \ll(U V)^{1+\varepsilon}
$$

for all $\alpha$ of type 1 , which completes the proof of the Lemma.

Lemma 6. For all $\alpha$ of type 1, we have

$$
S_{3}(x) \ll x^{1 / 4} V^{3 / 4}(\log x)^{2} .
$$

Proof. For any real $\alpha$ and $\beta$, let

$$
J=\sum_{m=1}^{t} \mathbf{e}\left(\alpha m^{2}+\beta m\right)
$$


Then, (see for example, [ECT, p. 99]), we have

$$
\begin{aligned}
|J|^{2} & \leq t+2 \sum_{j=1}^{t-1} \min (t,|\operatorname{cosec}(2 \pi \alpha j)|) \\
& \leq t+2 \sum_{j=1}^{t-1}\|j \alpha\|^{-1} \\
& \ll t^{1+\varepsilon}
\end{aligned}
$$

for all $\alpha$ of type 1 , by Lemma 3. Thus,

$$
\begin{aligned}
S_{3}(x) & =\sum_{n \leq x}\left(\sum_{\substack{d^{2} e=n \\
e \leq V}} \mu(e)\right) \mathbf{e}\left(d^{2} e \alpha\right) \\
& =\sum_{e \leq V} \mu(e) \sum_{d \leq \sqrt{x / e}} \mathbf{e}\left(d^{2} e \alpha\right) .
\end{aligned}
$$

By the penultimate estimate, we deduce that

$$
S_{3}(x) \ll \sum_{e \leq V} \frac{x^{1 / 4} \log ^{2} x}{e^{1 / 4}} \ll x^{1 / 4} V^{3 / 4}(\log x)^{2},
$$

for all $\alpha$ of type 1 . This completes the proof of the lemma.

Lemma 7. For all $\alpha$ of type 1, we have

$$
S_{4}(x) \ll x^{1+\varepsilon}\left(U^{-1 / 2}+V^{-1 / 2}\right) .
$$

Proof. We have

$$
S_{4}(x)=-\sum_{\substack{d e=n \leq x \\ d>U, e>V}} \lambda(d) f_{1}(e, V) \mathbf{e}(d e \alpha)
$$

where

$$
\left|f_{1}(e, V)\right|=\left|\sum_{\substack{t r=e \\ r \leq V}} \mu(r)\right| \leq d_{2}(e),
$$

the number of divisors of $e$. Let

$$
J_{1}=\sum_{\substack{W<d<2 W \\ e>V, d e \leq x}} \lambda(d) f_{1}(e, V) \mathbf{e}(d e \alpha),
$$


where $W \geq U$. By Cauchy-Schwarz inequality, we obtain

$$
\left|J_{1}\right| \leq\left(\sum_{\begin{array}{c}
W<d<2 W \\
d \leq x / V
\end{array}} \lambda(d)^{2}\right)^{1 / 2}\left(\sum_{\begin{array}{c}
W<d<2 W \\
d \leq x / V
\end{array}}\left|\sum_{\substack{e>V, e<x / d, e<x / W}} f_{1}(e, V) \mathbf{e}(d e \alpha)\right|^{2}\right)^{1 / 2}
$$

which we find easily to be

$$
\ll W^{1 / 2}\left(\sum_{\substack{W<d<2 W \\ d \leq x / V}} \sum_{\substack{V<e_{1}<x / d, x / W \\ V<e_{2}<x / d, x / W}} f_{1}\left(e_{1}, V\right) \overline{f_{1}\left(e_{2}, V\right)} \mathbf{e}\left(\left(e_{1}-e_{2}\right) d \alpha\right)\right)^{1 / 2} .
$$

The sum corresponding to $e_{1}=e_{2}$ contributes a quantity to the right hand side which does not exceed in absolute value

$$
W^{1 / 2}\left(\sum_{\begin{array}{l}
W<d<2 W \\
d \leq x / V
\end{array}} \sum_{V<e<x / d, x / W} d_{4}(e)\right)^{1 / 2}
$$

where $d_{4}(e)$ denotes the number of 4-tuples of positive integers whose product is $e$. By a well-known estimate [ECT, p. 313], this is easily seen to be

$$
\ll W^{1 / 2}\left(\frac{x^{2}}{V W} \log ^{3} \frac{x}{W}\right)^{1 / 2} \ll \frac{x(\log x)^{3 / 2}}{\sqrt{V}}
$$

For $e_{1} \neq e_{2}$, and fixed $j$, the equation $e_{1}-e_{2}=j$ determines a unique solution $e_{2}$ for every fixed $e_{1}$. Hence the sum corresponding to $e_{1} \neq e_{2}$ contributes a quantity to the right hand side of (5) which does not exceed in absolute value

$$
W^{1 / 2} x^{\varepsilon}\left(\sum_{j \leq x / W} \frac{j}{\|j \alpha\|}\right)^{1 / 2}
$$

which is, by Lemma 3, bounded by

$$
x^{1+2 \varepsilon} / \sqrt{W}
$$

for all $\alpha$ of type 1 . Combining all the estimates, we obtain

$$
\left|J_{1}\right| \ll \frac{x}{\sqrt{V}}(\log x)^{3 / 2}+\frac{x^{1+2 \varepsilon}}{\sqrt{W}}
$$

Now we take $W=2^{t} U$, where $t=0,1,2, \ldots\left[\log _{2}(x / U V)\right]$ which runs over $O(\log x)$ intervals so that the result follows from the above estimate. 


\section{Proof of Theorem 1}

From Lemmas 3 to 6, we see that

$$
S(x, \alpha) \ll U+(U V)^{1+\varepsilon}+x^{1 / 4+\varepsilon} V^{3 / 4}+x\left(\frac{1}{\sqrt{U}}+\frac{1}{\sqrt{V}}\right) x^{\varepsilon}
$$

for all $\alpha$ of type 1 . We choose $U=V=x^{2 / 5}$ to obtain a final estimate of $x^{4 / 5+\varepsilon}$ as desired.

\section{Proof of Theorem 2}

The method used above to treat the sum

$$
\sum_{n \leq x} \lambda(n) \mathbf{e}(n \alpha)
$$

can also be used to treat more general sums.

As before, let us set,

$$
\begin{aligned}
& A(s)=\sum_{n=1}^{\infty} \frac{a(n)}{n^{s}} \quad B(s)=\sum_{n=1}^{\infty} \frac{b(n)}{n^{s}} \\
& \frac{A(s)}{B(s)}=\sum_{n=1}^{\infty} \frac{c(n)}{n^{s}} \quad \frac{1}{B(s)}=\sum_{n=1}^{\infty} \frac{\tilde{b}(n)}{n^{s}} \\
& F(s)=\sum_{n \leq U} \frac{c(n)}{n^{s}} \quad G(s)=\sum_{n=1}^{\infty} \frac{\tilde{b}(n)}{n^{s}} .
\end{aligned}
$$

We assume that $a(n), b(n), \tilde{b}(n)$ satisfy an estimate of the type $O\left(n^{\varepsilon}\right)$ for any $\varepsilon>0$. We suppose that for all $\alpha$ of type 1 ,

$$
\sum_{n \leq x} a(n) \mathbf{e}(n \alpha) \ll x^{\theta} \min \left(1, \frac{1}{\|\alpha\|}\right)
$$

for some $\theta<1$ and

$$
\sum_{n \leq x} b(n) \mathbf{e}(n \alpha) \ll x^{\phi} \min \left(1, \frac{1}{\|\alpha\|}\right)
$$

for some $0 \leq \phi<1$. Then, there is a $\psi<1$ such that

$$
T(x):=\sum_{n \leq x} c(n) \mathbf{e}(n \alpha) \ll x^{\psi} \min \left(1, \frac{1}{\|\alpha\|}\right)
$$


for all $\alpha$ of type 1 . We will only give a brief indication of the proof since it follows the line of thought similar to the proof of Theorem 1 . We have by Lemma 1,

$$
c(n)=a_{1}(n)+a_{2}(n)+a_{3}(n)+a_{4}(n)
$$

and the corresponding sums

$$
S_{i}(x)=\sum_{n \leq x} a_{i}(n) \mathbf{e}(n \alpha) .
$$

Clearly, $\left|S_{1}\right| \leq U^{1+\varepsilon}$. Also,

$$
S_{2}(x)=-\sum_{t \leq U V}\left(\sum_{\substack{e r=t \\ e \leq U, r \leq V}} c(e) \tilde{b}(r)\right) \sum_{d \leq x / t} b(d) \mathbf{e}(d t \alpha),
$$

which by (7) gives

$$
S_{2}(x) \ll x^{\phi}(U V)^{1-\phi+\varepsilon} .
$$

For $S_{3}(x)$, we have by (6),

$$
S_{3}(x)=-\sum_{e \leq V} \tilde{b}(e) \sum_{d \leq x / e} a(d) \mathbf{e}(d \alpha) \ll x^{\theta} V^{1-\theta+\varepsilon} .
$$

Finally,

$$
S_{4}(x)=\sum_{\substack{d e=n \leq x \\ d>U, e>V}} c(d) f(e, V) \mathbf{e}(d e \alpha)
$$

where

$$
f(e, V)=\sum_{\substack{t r=e \\ r \leq V}} b(t) \tilde{b}(r)
$$

As before, we break this sum into dyadic intervals:

$$
J_{2}=\sum_{\substack{W<d<2 W \\ e>V, d e \leq x}} c(d) f(e, V) \mathbf{e}(d e \alpha)
$$

so by Cauchy-Schwarz, we get

$$
\left|J_{2}\right| \leq W^{1 / 2+\varepsilon}\left(\sum_{\substack{W<d<2 W \\ d<x / V}}\left|\sum_{\substack{e>V \\ e<x / d, x / W}} f(e, V) \mathbf{e}(d e \alpha)\right|^{2}\right)^{1 / 2}
$$


which we find satisfies

$$
\left|J_{2}\right| \ll W^{1 / 2+\varepsilon}\left(\sum_{\substack{W<d<2 W \\ d \leq x / V}} \sum_{\substack{V<e_{1}<x / d, x / W \\ V<e_{2}<x / d, x / W}} f\left(e_{1}, V\right) \overline{f\left(e_{2}, V\right)} \mathbf{e}\left(\left(e_{1}-e_{2}\right) d \alpha\right)\right)^{1 / 2} .
$$

The sum corresponding to $e_{1}=e_{2}$ gives a contribution

$$
W^{1 / 2+\varepsilon}\left(\sum_{\substack{W<d<2 W \\ d \leq x / V}}(x / W)^{1+2 \varepsilon}\right)^{1 / 2} \ll x^{1+\varepsilon} V^{-1 / 2}
$$

For $e_{1} \neq e_{2}$, the contribution is $x^{1+\varepsilon} W^{-1 / 2}$ as before. Combining these estimates gives

$$
T(x) \ll U^{1+\varepsilon}+x^{\phi}(U V)^{1-\phi+\varepsilon}+x^{\theta} V^{1-\theta+\varepsilon}+x^{1+\varepsilon}\left(U^{-1 / 2}+V^{-1 / 2}\right) .
$$

We choose $U=V$ to get

$$
T(x) \ll x^{\phi} V^{2-2 \phi+\varepsilon}+x^{\theta} V^{1-\theta+\varepsilon}+x^{1+\varepsilon} V^{-1 / 2}+V^{1+\varepsilon} .
$$

Since $\theta, \phi<1$, we see that for $V=x^{\delta}$ for some sufficiently small $\delta$, we get the desired result.

\section{Proof of Theorem 3}

In (8), we set $\phi=0$ since

$$
\sum_{n \leq x} \mathbf{e}(n \alpha) \ll \min \left(1, \frac{1}{\|\alpha\|}\right)
$$

The corresponding $T(x)$ is

$$
\ll V^{2+\varepsilon}+x^{\theta} V^{1-\theta+\varepsilon}+V^{-1 / 2} x^{1+\varepsilon} .
$$

Now set $V^{2+\varepsilon}=V^{-1 / 2} x^{1+\varepsilon}$ so that $V^{5 / 2+\varepsilon}=x^{1+\varepsilon}$. This gives the stated estimate.

One can derive a slightly more precise result by making the following observation. From inequality (8), we have

$$
T(x) \ll x^{\phi} V^{2-2 \phi+2 \varepsilon}+x^{\theta} V^{1-\theta+\varepsilon}+x^{1+\varepsilon} V^{-1 / 2}+V^{1+\varepsilon} .
$$

We choose $V$ such that $x^{\theta} V^{1-\theta}=x V^{-1 / 2}$ so that $V=x^{2(1-\theta) /(3-2 \theta)}$. Hence,

$$
T(x) \ll x^{\phi+4(1-\theta)(1-\phi) /(3-2 \theta)+2 \varepsilon}+x^{\theta+2(1-\theta)^{2} /(3-2 \theta)+2 \varepsilon} .
$$


Note that if $\theta>1 / 2$, then

$$
\phi+\frac{4(1-\theta)(1-\phi)}{3-2 \theta}<1
$$

and

$$
\theta+\frac{2(1-\theta)^{2}}{3-2 \theta}<1
$$

So we can take in this case, any $\psi$ satisfying

$$
\psi>\max \left\{\phi+\frac{4(1-\theta)(1-\phi)}{3-2 \theta}, \theta+\frac{2(1-\theta)^{2}}{3-2 \theta}\right\} .
$$

In the case $2 \phi<\theta<1$, we choose $V$ such that

$$
x^{\phi} V^{2-2 \phi}=x^{\theta} V^{1-\theta}
$$

which allows us to take any $\psi$ satisfying the inequality

$$
\psi>\max \left\{\phi+\frac{2(1-\phi)(\theta-\phi)}{1+\theta-2 \phi}, 1-\frac{\theta-\phi}{2(1+\theta-2 \phi)}\right\}
$$

\section{Proofs of the corollaries}

To deduce Corollary 4, we let $A(s)=1, B(s)=\zeta(s)$. To deduce Corollary 5, we let $A(s)=-\zeta^{\prime}(s)$ and $B(s)=\zeta(s)$. We must estimate

$$
\sum_{n \leq x}(\log n) \mathbf{e}(n \alpha)
$$

But this is easily done by partial summation:

$$
\sum_{n \leq x}(\log n) \mathbf{e}(n \alpha) \ll(\log x) \min \left(1, \frac{1}{\|\alpha\|}\right) .
$$

Therefore, we can take $\theta=\varepsilon$ to deduce the result.

\section{References}

[AS] Anderson, R., Stark, H. M.: Oscillation theorems. In: Analytic Number Theory (Philadelphia, Pa., 1980), pp. 79-106. Lecture Notes in Mathematics 899 Springer, BerlinNew York 1981

[BH] Baker, R. C., Harman, G.: Exponential sums formed with the Möbius function. Journal of the London Math. Soc. (2) 43 (1991), 193-198 
[BC] Bateman, P., Chowla, S.: Some special trigonometrical series related to the distribution of prime numbers. Journal of the London Math. Society 38 (1963), 372-374

[C] Carleson, L.: On convergence and growth of partial sums of Fourier series. Acta Math. 116 (1966), 135-157

[CN] Chandrasekharan, K., Narasimhan, R.: Exponential sums associated with the Dedekind zeta function. Comment. Math. Helv. 52 (1977), 49-87

[Ch] Chowla, S.: Some problems of diophantine approximation I. Math. Zeit. 33 (1931), $544-563$

[DD] Daboussi, H., Delange, H.: On multiplicative arithmetical functions whose modulus does not exceed one. J. London Math. Soc. (2) 26 (1982), 245-264

[D] Davenport, H.: On some infinite series involving arithmetical functions. Quart. J. Math., Oxford Ser. (2) 8 (1937), 313-320

[Da] Davenport, H.: Multiplicative Number Theory, 2nd Edition (edited by H. Montgomery). Springer-Verlag

[De] Delange, H.: Generalisation of Daboussi's theorem. In: Topics in Classical Number Theory, Vol. 1 and 2 (Budapest, 1981) p. 305-318. Colloq. Math. Soc. János Bolyai 34. North Holland, Amsterdam, New York 1984

[E] Erdös, P.: Some remarks on Diophantine approximations. J. Indian Math. Soc. 12 (1948), 67-74

[EM] Esmonde, J., Ram Murty, M.: Problems in Algebraic Number Theory. Grad. Text. Math. 190. Springer-Verlag, 1998

[G] Goubin, L.: Sommes d'exponentielles et principe de l'hyperbole. Acta Arith. 73 (1995), 303-324

[HS] Hajela, D., Smith, B.: On the maximum of an exponential sum of the Möbius function. Lecture Notes in Mathematics 1240 (1987), 145-164

[H] Haselgrove, C. B.: A disproof of a conjecture of Pólya. Mathematika 5 (1958), 141-145

[KN] Kuipers. L., Niederreiter, H.: Uniform Distribution of Sequences: Wiley, 1974

[La] Lang, S.: Introduction to Diophantine Approximations. Addison-Wesley, 1966

[L] Lehman, R.: On Liouville's function. Math. Comp. 14 (1960), 311-320

[MV] Montgomery, H., Vaughan, R. C.: Exponential sums with multiplicative coefficents. Inventiones Math. 43 (1977), 69-82

[Mu Murty, R.: Some remarks on the Riemann hypothesis; to appear in the Bombay Proceedings of the conference on $L$-functions and Cohomology of Arithmetic Groups, Tata Institute, January, 1999

[P] Pólya, G.: Verscheidene Bemerkungen zur Zahlentheorie. Jahresbericht der deutschen Math.-Vereinigung 28 (1919), 31-40

[ECT] Titchmarsh, E. C.: The theory of the Riemann zeta function, revised by D. R. HeathBrown (1986). Clarendon Press, Oxford

Received revised June, 2000

M. Ram Murty, Department of Mathematics, Queen's University, Kingston, Ontario, K7L 3N6, Canada murty@mast.queensu.ca

A. Sankaranarayanan, School of Mathematics, Tata Institute of Fundamental Research, Homi Bhabha Road, Mumbai, 400 005, India sank@math.tifr.res.in 